Historic, Archive Document

Do not assume content reflects current scientific knowledge, policies, or practices. 



\title{
68.39
}

\section{Suggestions for Fall Planting}

\section{Gillett's Ferm and Flower Farm}

\author{
K. E. Gillett, Proprietor \\ SOUTHWICK, MASSACHUSETTS
}

Herewith I am submitting suggestions for Fall planting. Most native plants can be successfully moved in the Fall. There is a group of American plants that should preferably be planted in the Autumn because they start making their growth as soon as the frost leaves the ground and to move them when in growth means a set-back for that season.

Some of the best of that group follow.

On all items herein listed five will be sold at the ten rate;

fifty at the hundred rate.

Arisaema triphyllum (Jack-in-the-Pulpit)

Claytonia virginica (Spring Beauty)

\begin{tabular}{ccr} 
Each & 10 & \multicolumn{1}{c}{100} \\
$25 \mathrm{c}$ & $\$ 1.50$ & $\$ 12.00$ \\
$15 \mathrm{c}$ & 1.00 & 8.00 \\
$15 \mathrm{c}$ & 1.00 & 8.00 \\
$15 \mathrm{c}$ & 1.00 & 8.00 \\
$15 \mathrm{c}$ & 1.00 & 8.00 \\
$15 \mathrm{c}$ & 1.00 & 8.00 \\
$15 \mathrm{c}$ & 1.00 & 8.00 \\
$15 \mathrm{c}$ & 1.00 & 8.00 \\
$15 \mathrm{c}$ & 1.00 & 8.00 \\
$25 \mathrm{c}$ & 1.50 & 12.00 \\
$25 \mathrm{c}$ & 1.50 & 12.00 \\
$25 \mathrm{c}$ & 2.00 & 15.00 \\
$25 \mathrm{c}$ & 2.00 & 15.00 \\
$25 \mathrm{c}$ & 2.00 & 15.00 \\
$25 \mathrm{c}$ & 1.50 & 12.00 \\
$15 \mathrm{c}$ & 1.00 & 8.00 \\
$15 \mathrm{c}$ & 1.00 & 8.00 \\
$15 \mathrm{c}$ & 1.00 & 8.00 \\
$15 \mathrm{c}$ & 1.00 & 6.00 \\
$20 \mathrm{c}$ & 1.25 & 10.00 \\
$15 \mathrm{c}$ & 1.00 & 6.00 \\
\hline $12 \mathrm{y}$
\end{tabular}

Dicentra Cucullaria (Dutchman's Breeches)

Dicentra canadensis (Squirrel Corn)

Erythronium americanum (Adder's Tongue)

Erythronium americanum albidum (Flowers white)

Erythronium-citrinum (Flowers light yellow)

Erythronium grandiflorum (Deep yellow)

Erythronium Hendersoni (Flowers purple)

Hepatica acutiloba

Hepatica triloba

Lilium canadense (Meadow Lily)

Lilium philadelphicum (Turk's Cap Lily)

Lilium superbum (Superb Lily)

Mertensia virginica (Virginia Bluebell)

Thalictrum anemonoides (Rue Anemone)

Trillium erectum (Red Trillium)

Trillium erythrocarpum (Painted Trillium)

Trillium grandiflorum (White Trillium)

Trillium stylosum (Pink Trillium)

Sanginaria canadensis (Bloodroot)

An Illustrated Descriptive Catalogue will be mailed upon request. 


\section{PLANT FERNS IN THE FALL.}

I am herewith submitting my complete list of ferns all of which can be successfully planted in the autumn:

Adiantum pedatum (Maidenhair Fern)

Aspidium acrostichoides (Christmas Fern)

Aspidium aculeatum Braunii (Prickly Shield Fern)

Aspidium cristatum (Crested Wood Fern)

Aspidium cristatum Clintonianum

Aspidium Goldieanum (Goldie's Wood Fern)

Aspidium marginale (Evergreen Wood Fern)

Aspidium noveboracense (N. Y. Shield Fern)

Aspidium spinulosum (Spinulose Wood Fern)

Aspidium spinulosum dilatatum

Aspidium Thelypteris (Marsh Shield Fern)

$\begin{array}{lrr}\text { Each } & 10 & 100 \\ 25 \mathrm{c} & \$ 1.50 & \$ 12.00 \\ 25 \mathrm{c} & 1.50 & 12.00 \\ 40 \mathrm{c} & 3.50 & 30.00 \\ 25 \mathrm{c} & 1.50 & 12.00 \\ 25 \mathrm{c} & 1.50 & 12.00 \\ 50 \mathrm{c} & 4.50 & 40.00 \\ 25 \mathrm{c} & 1.50 & 12.00 \\ 25 \mathrm{c} & 1.50 & 12.00 \\ 25 \mathrm{c} & 1.50 & 12.00 \\ 30 \mathrm{c} & 2.00 & 15.00 \\ 25 \mathrm{c} & 1.50 & 12.00 \\ 30 \mathrm{c} & 2.50 & 20.00 \\ 25 \mathrm{c} & 1.50 & 12.00 \\ 25 \mathrm{c} & 1.50 & 12.00 \\ 25 \mathrm{c} & 1.50 & 12.00 \\ 25 \mathrm{c} & 1.50 & 12.00 \\ 25 \mathrm{c} & 1.50 & 12.00 \\ 25 \mathrm{c} & 1.50 & 12.00 \\ 25 \mathrm{c} & 1.50 & 12.00\end{array}$

$\begin{array}{llll}\text { Asplenium angustifolium (Narrow-leaved Spleenwort) } & 30 \mathrm{c} & 2.50 & 20.00 \\ \text { Asplenium ebeneum (Ebony Spleenwort) } & 25 \mathrm{c} & 1.50 & 12.00 \\ \text { Asplenium Filix-foemina (Lady Fern) } & 25 \mathrm{c} & 1.50 & 12.00 \\ \text { Asplenium Trichomanes (Maidenhair Spleenwort) } & 25 \mathrm{c} & 1.50 & 12.00 \\ \text { Asplenium thelypteroides (Silvery Spleenwort) } & 25 \mathrm{c} & 1.50 & 12.00 \\ \text { Cystopteris bulbifera (Bladder Fern) } & 25 \mathrm{c} & 1.50 & 12.00 \\ \text { Cystopteris fragilis } & 25 \mathrm{c} & 1.50 & 12.00 \\ \text { Dicksonia punctilobula (Hay-scented Fern) } & 25 \mathrm{c} & 1.50 & 12.00\end{array}$

Dicksonia punctilobula $\quad 60 \mathrm{c}$ per sq. ft.; $\$ 5.00$ per 10 sq. ft.;

Onoclea sensibilis (Sensitive Fern)

$\$ 35.00$ per 100 sq. $\mathrm{ft}$.

Onoclea Struthiopteris (Ostrich Fern)

Osmunda regalis (Flowering Fern)

$25 \mathrm{c} \quad 1.50 \quad 12.00$

Osmunda Claytoniana (Interrupted Fern)

$25 \mathrm{c} \quad 2.00 \quad 15.00$

Osmunda cinnamonea (Cinnamon Fern)

$25 \mathrm{c} \quad 2.00 \quad 15.00$

Phegopteris Dryopteris (Beech Fern)

Phegopteris hexagonoptera

Phegopteris polypodioides

Polypodium vulgare (Common Polypody)

Pteris aquilina (Common Brake)

Woodsia ilvensis (Rusty Woodsia)

Woodsia obtusa (Obtuse-leaved Woodsia)

Woodwardia angustifolia (Chain Fern)

Woodwardia virginica

$\begin{array}{lrr}25 \mathrm{c} & 2.00 & 15.00 \\ 25 \mathrm{c} & 2.00 & 15.00 \\ 25 \mathrm{c} & 1.50 & 12.00 \\ 25 \mathrm{c} & 1.50 & 12.00 \\ 25 \mathrm{c} & 1.50 & 12.00 \\ 25 \mathrm{c} & 1.50 & 12.00 \\ 25 \mathrm{c} & 1.50 & 12.00 \\ 25 \mathrm{c} & 1.50 & 12.00 \\ 25 \mathrm{c} & 1.50 & 12.00 \\ 25 \mathrm{c} & 2.00 & \\ 25 \mathrm{c} & 1.50 & 12.00\end{array}$

\section{PLANTS FOR ROCK GARDENS}

I have a good collection of rock garden plants for delivery this Autumn, and am herewith making special offer as follows:

100 choice rock garden plants in 10 varieties for $\$ 10.00$

50 choice rock garden plants in 10 varieties for 5.50

For complete list consult my catalogue which was mailed you earlier in the season. 


\section{AZALEAS AND BROADLEAVED EVERGREENS}

Do not put off the planting of Azaleas and Broadleaved Evergreens until Spring if you can conveniently plant this Autumn. All Azaleas and Broadleaved Evergreens are dug with ball of earth, securely burlapped and packed, and delivered to the local express or freight office at the prices quoted.

Azalea arborescens (Sweet Azalea) 1-11/2' Azalea calendulacea (Flame Azalea) 1-1 $1 \mathbf{2}^{\prime}$ Azalea calendulacea $11 / 2-2^{\prime}$ Each $\quad 10 \quad 100$ Azalea calendulacea 2-21/2'

Azalea canescens (Piedmont Azalea) 1-1 1/2'

$\begin{array}{rrr}\$ 1.75 & \$ 15.00 & \\ 2.50 & 20.00 & \$ 175.00 \\ 3.00 & 25.00 & 225.00 \\ 3.60 & 33.00 & \\ 2.00 & 17.50 & 150.00 \\ 3.00 & 25.00 & 225.00 \\ 1.75 & 15.00 & 125.00 \\ 1.50 & 12.50 & 100.00 \\ 1.65 & 15.00 & 135.00 \\ 2.50 & 22.50 & 200.00\end{array}$

Azalea canescens (Piedmont Azalea) 11/2-2'

Azalea nudiflora (Pinxter Bloom) 1-1 1/2'

Azalea viscosa (Swamp Azalea) 11/2-2'

Azalea viscosa (Swamp Azalea) 2-21/2'

Azalea viscosa (Swamp Azalea) 21/2-3'

Andromeda floribunda (Lily of the Valley Shrub)

$12-15^{\prime \prime}$

$15-18^{\prime \prime}$

2.75

25.00

225.00

$18-24^{\prime \prime}$

3.50

32.50

300.00

Calluna vulgaris (Heather) 8-10"

4.50

Calluna vulgaris (Heather) 10-12"

1.50

12.50

100.00

2.00

15.00

125.00

Daphne cneorum 6-8"

.75

6.50

60.00

Daphne cneorum 8-10"

1.00

Daphne cneorum 10-12"

1.25

9.00

85.00

Daphne cneorum 12-15"

2.00

11.50

110.00

Ilex glabra (Inkberry) 12-15"

2.00

17.50

160.00

Ilex glabra (Inkberry) 15-18"

2.50

150.00

Ilex glabra (Inkberry) 18-24"

Kalmia latifolia (Mountain Laurel) 12-15"

3.00

17.50

1.20

27.00

Kalmia latifolia (Mountain Laurel) 15-18"

1.60

10.00

90.00

Kalmia latifolia (Mountain Laurel) 18-24"

2.50

15.00

125.00

Leucothoe catesbaei (Drooping Leucothoe) 12-15"

1.00

22.50

200.00

Leucothoe catesbaei (Drooping Leucothoe) 15-18"

1.50

8.50

75.00

Rhododendron carolinianum (Carolina Rhododendron)

$$
\begin{gathered}
9-12^{\prime \prime} \\
12-15^{\prime \prime} \\
15-18 \prime \prime \\
18-24^{\prime \prime}
\end{gathered}
$$

100.00

2.75

3.25

25.00

150.00

225.00

$4.00 \quad 37.50$

275.00

Rhododendron catawbiense (Catawba Rhododendron) $1-1 \frac{1}{2}$

Rhododendron maximum 1-11/2'

$2.00 \quad 17.50$

160.00

$1.25 \quad 11.50$

100.00

Rhododendron maximum 11/2-2'

2.00

17.50

160.00

Phododendron maximum 2-21/2'

$2.75 \quad 25.00$

Rhododendron maximum 1-11/2' extra broad specimens $1.75 \quad 15.00$

Rhododendron maximum $1 \frac{1}{2}-2^{\prime}$ extra broad specimens

$3.00 \quad 27.50$ 


\section{CONIFEROUS EVERGREENS}

Prices f. o. b. Southwick, burlapping and packing charges are included.

Each 10

Thuya occidentalis (American Arborvitae) $1 \frac{112-2^{\prime}}{\$ 1.50} \quad \$ 12.50$

Thuya occidentalis (American Arborvitae) 2-2 $1 \mathbf{2}^{\prime} \quad 2.25 \quad 20.00$

Thuya occidentalis (American Arborvitae) 21/2-3' $3.00 \quad 27.50$

Thuya occidentalis (American Arborvitae) $3-4^{\prime} \quad 4.00 \quad 35.00$

Thuya occidentalis Pyramidalis (Pyramidal Arborvitae)
$2-21 / 2^{\prime}$
$3.00 \quad 25.00$
$21 / 2-3^{\prime}$
$4.00 \quad 35.00$
Tsuga canadensis (Common Hemlock) 1-11/2' 。 $\quad 1.75 \quad 15.00$
Tsuga canadensis (Common Hemlock) $1 \frac{1 / 2-2^{\prime}}{2.50} 22.50$
Tsuga canadensis (Common Hemlock) $2-3^{\prime} \quad 3.50 \quad 30.00$

\section{GRANULATED PEAT MOSS}

Azaleas, Rhododendrons, and similar plants need an acid soil. This can be supplied in a great degree by Granulated Peat Moss worked into the soil and used as a mulch. The price is $\$ 4.00$ a bale f. o. b. Southwick, Mass., Boston, Mass., or New York City. A bale will cover 240 square feet, one inch deep.

\section{Gillett's Fern and Flower Farm}

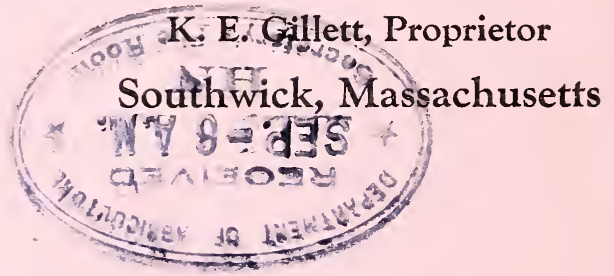

